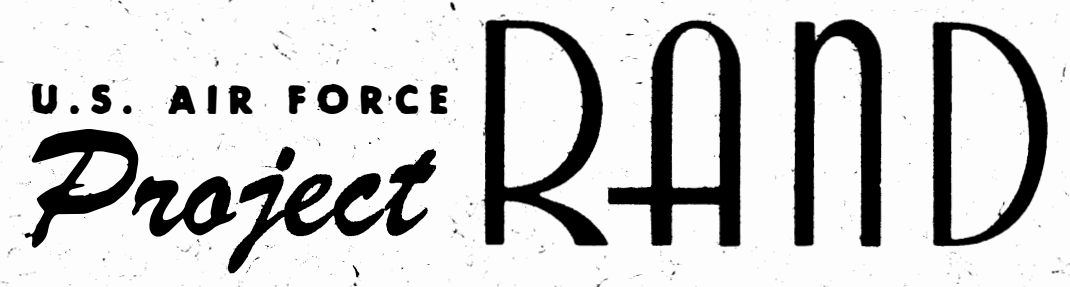

MIXING IN INHOMOGENOUS GAS JETS

BATTELLE MEMORIAL INSTITUTE - COLUMBUS, OHIO

February, 1949

$R-142$ 


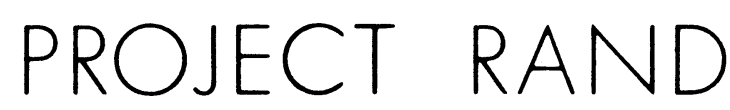

\title{
MIXING IN INHOMOGENOUS GAS JETS
}

\author{
W. R. Keagy - A. E. Weller \\ F. A. Reed - W. T. Reid
}

battelle meMORIAL INSTITUTE - COLUMBUS. OHIO

February, 1949

R- 142

COPY NO. 


.

, 


\section{CONTENTS}

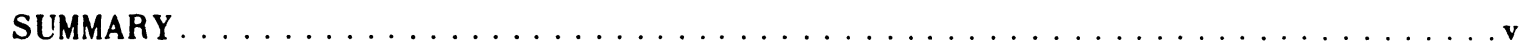

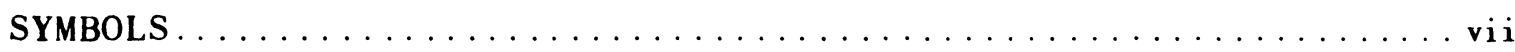

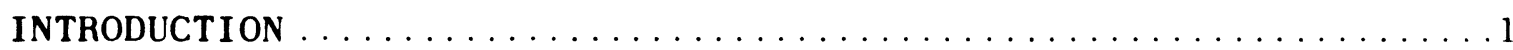

MIXING IN FREELY EXPANDING GAS JETS $\ldots \ldots \ldots \ldots \ldots \ldots \ldots \ldots \ldots \ldots$

THEORY OF A FREELY EXPANDING INHOMOGENEOUS JET $\ldots \ldots \ldots \ldots \ldots \ldots \ldots 4$

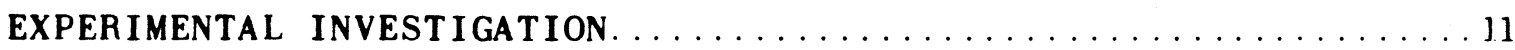

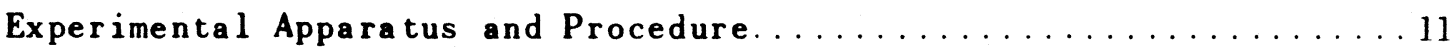

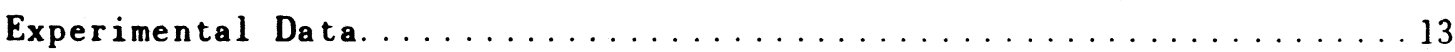

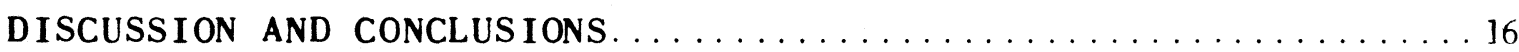



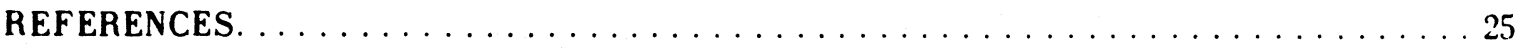

\section{FIGURES}

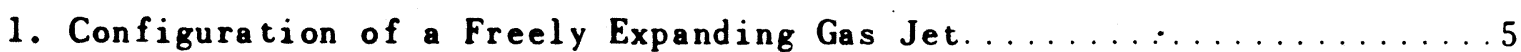

2. Experimental Apparatus for Collecting Samples of Gas............12

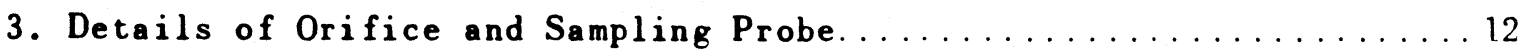

4. Comparison of Measured Profiles in Jet of Nitrogen

With the Calculated Values......................... 13

5. Comparison of Measured Profiles in a Jet of Carbon

Dioxide With the Calculated Values................... 13

6. Comparison of Measured Profiles in a Jet of Helium

With the Calculated Values.......................... 15 
7. Comparison of Measured Axial Concentration and Velocity

in a Jet of Nitrogen With the Calculated Values............. 15

8. Comparison of Measured Axial Concentration and Velocity

in a Jet of Carbon Dioxide With the Calculated Values............15

9. Comparison of Measured Axial Concentration and Velocity

in a Jet of Helium With the Calculated Values................. 5 


\section{SUMMARY}

A discussion of the problem of a freely expanding turbulent jet is presented and some of the more significant work which has been done on this and similar problems is described briefly. Although a complete solution of the general equations of flow in a freely expanding inhomogeneous jet is not now available, yet approximate expressions may be obtained for the values of the mean concentration and mean velocity along the axis of the jet. This can be done by assuming the velocity and concentration profiles to be similar at all cross sections and by approximating them with error functions. Preliminary experimental data from jets of helium, nitrogen, and carbon dioxide obtained by microsampling and by measurement of impact pressure give evidence of the assumed similarity of the profiles of concentration and velocity. Although the data are not entirely consistent among themselves, the agreement between the measured values of mean axial concentration and those predicted by the analysis is quite satisfactory. The results show the mean concentration at any point in the jet to vary inversely as the density of the jet fluid and show the mean velocity to vary directly with the density of the jet fluid. The effect of buoyancy on the concentration is predicted, but the experimental data are not sufficiently extensive to confirm the predictions. 


\section{SYMBOLS}

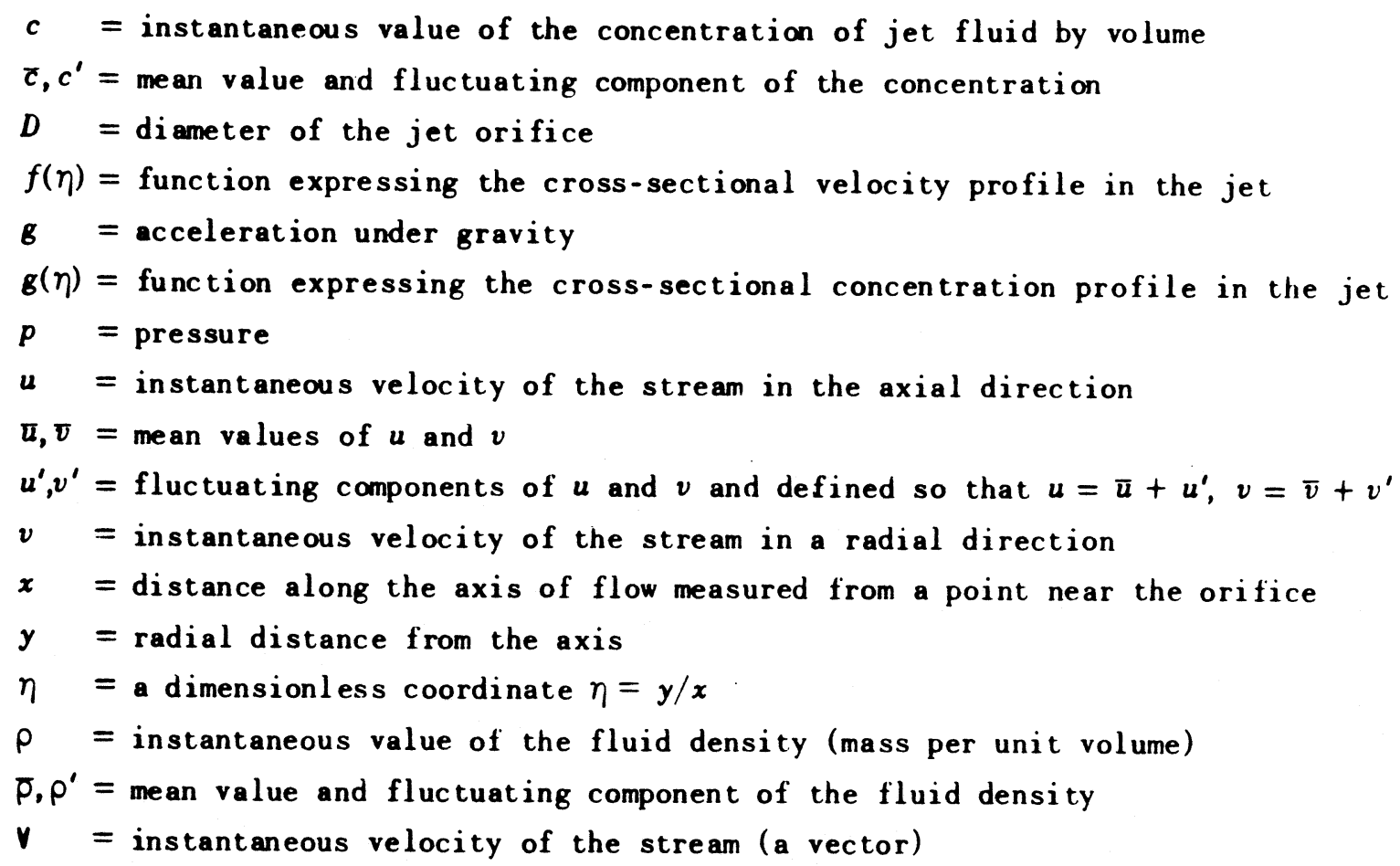

\section{Sabscripts}

- refers to values at orifice

$m$ refers to values along the axis

$s$ refers to values outside the jet (i.e., $P_{s}$ is the density of the surroundings; in this case, air) 


\title{
MIXING IN INHOMOGENEOUS GAS JETS
}

\author{
I N T ROD UCT I O N
}

The importance of mixing as it occurs in the combustion process in rocket and jet motors cannot yet be fully determined because of a general lack of understanding of the fundamental principles involved in this process. It is reasonable to imagine a simplified mechanism of such combustion as a successive occurrence of the processes of injection, mixing, ignition, and burning, each of which may be studied independently with results which will benefit the interpretation of any combination of these processes. If such reasoning is sound, then the injection and mixing process is seen to be unique, in that, for any given combination of propellants burning under operating conditions which are determined by performance specifications, it is the only step in the combustion process which may be controlled to any extent by purely mechanical considerations. This consideration assumes increasing importance as higher propellant flow rates are utilized and as structural limitations prohibit corresponding increase of the combustion space.

The importance of the mixing step in problems of heat transfer is already recognized. It need only be pointed out that film cooling, extensively employed in rocket design since the German V-2, represents one very successful way in which "controlled mixing" can be used to reduce heat transfer to the walls of the combustion chamber and nozzle of the rocket. The extent to which temperature distribution in the combustion chamber may be affected by the mixing pattern, however, remains to be answered by a more complete investigation.

The fact that it has been possible to construct and operate rocket motors which provide reliable performance and achieve satisfactory combustion efficiency is not evidence of complete understanding of the mixing process, nor does it necessarily argue that, under other operating conditions, a better understanding of the mixing step might not be essential to successful design. The basis for present designs of propellant injectors in rocket motors is almost wholly empirical. Measurements of heat transfer and thrust in large number of tests have led to the development of successful motors. Inspired guesswork, aided by water-spray tests, has been successful in leading to improvements in injector design. The observation of hot spots and the occurrence of structural failure have enabled the development of adequate and reliable cooling systems. Through all of this development, however, little has been added to the understanding of the basic principles involved. 
This situation can hardly be taken as a criticism of accomplishments to date. It is rather a credit to designers that their efforts have been so successful in - field where adequate analytic methods are so lacking. The problem of mixing as it occurs in acket motor is sufficiently complex that no satisfactory solution can be expected without a separate investigation of the effects of the several factors which serve to complicate it. These include the absence of symetry by continuous rotation, the occurrence of combustion in the mixing region, the presence of confining walls, the existence of thermal and density gradients, and the change of phase from liquid to gas in the mixing region.

Although some study of most of these factors has been made or is currently in progress, the only problem for which the analysis seems to any degree satisfactory is the comparatively simple one of a single freely expanding jet of fluid of the same density as the surroundings with which it mixes. If the jet is inhomogeneous, that is, if it consists of a fluid that differs in density from the surroundings, the values of the mean velocity and the mean concentration of the jet fluid will be affected by the density gradients. This report presents the results of a preliminary investigation of this effect in jets of nitrogen, helium, and carbon dioxide.

\section{MIXING IN FREELY EXPANDING GAS JETS}

The comparatively simple problem of mixing in single freely expanding jet is still sufficiently complicated that no solution based upon a physically sound theory of turbulence has yet been obtained. This situation would seem to offer little hope of achieving solutions to problems which are more complicated. It has been demonstrated, however, that physically sound theory of turbulence is not required to obtain useful results from the analysis, and this consideration has led to the development of a pseudotheory which, though essentially empirical, has been able to provide deeper insight into the problem of mixing in jets than might otherwise be possible.

All of the theories of turbulence which have been sufficiently developed to be of any practical use may be classed under two general types. The first of these, the statistical theories, are founded upon observed relationships between the turbulent fluctuations in the stream. These are found to be statistical in nature, and, together with increasing experimental results from measurements with hot-wire instruments, the theory has been able to establish certain statistical correlations among these fluctuating values. In problems where information regarding the fluctuations themselves is required, it is essential that the statistical approach be followed. In cases where it is necessary only to determine the effect of the turbulence upon the over-all flow conditions, however, this approach is far too complex to be very successful. 
The second of these, the phenomenological theories of turbulence, are much better suited to cases in which a detailed study of the turbulence itself is not required. These include the various theories in which an attempt is made to relate the turbulent fluctuations of the flow parameters to their average values through the use of one of several distance parameters called the mixing length. Since the mixing length has little physical significance, and its value is in no way specified in the theory, nothing is gained unless it can be shown that the mixing length varies in some simple way over the region of flow. Fortunately, this has been possible in the case of free jet, with result that enables the equations of motion and continuity to be expressed in terms of the mean values of the stream variables a lone. Thus, the jet problem is reduced to one of applied mathematics. In so doing, however, it should be pointed out that all hope of gaining information concerning the nature of the turbulence in the jet has been sacrificed.

The mixing-length theories have been used to derive analytical expressions for the mean values of the flow parameters in number of problems. Tollmien's(1) solution for the velocity in round jet with constant fluid density is a notable example; the analysis is presented in Appendix $I$. He assumed the mixing length, based on Prandtl's momentum transfer theory, to be proportional to the breadth of the jet and to be constant over any cross section. The resultant expression for the velocity profiles shows excellent agreement with measured values. Tomotika, (2) using a mixing length based on Taylor's vorticity transport hypothesis, (3) has shown that the resultant expression for the velocity is identical with that obtained by Tollmien, whereas the expression which it gives for temperature distribution in a heated jet is in better agreement with experiment than that obtained using the momentum transport hypothesis. Kuethe ${ }^{(4)}$ extended the analysis of Tollmien to the region of potential flow near the orifice, and Squire and Trouncer ( ${ }^{(8)}$ have successfully handled the problem of a jet in a coaxially moving air stream. Hawthorne ${ }^{(0)}$ emphasized the importance of these developments to the study of mixing by obtaining an expression for the mean concentration of the primary fluid at any point in the jet. His result, shown in Appendix $I$, is that which would be expected to follow from Taylor's expression for temperature on the basis of Reynolds' analogy between temperature and material transfer. Hawthorne also pointed out the relation between the angle subtended by the jet and the values of constant terms which appear in the expressions for the velocity and concentration.

Coincident with the foregoing developments, investigation of wakes behind bluff bodies in moving air streams revealed that this type of problem showed a general similarity to that of the freely expanding jet. Treatment of these by Swain ( 7$)$ and Schlichting ${ }^{(8)}$ followed Tollmien's original work by only a few years. Squire ${ }^{(\theta)}$ has recently summarized all of these developments in an excellent article that emphasizes the extent to which the analysis may be carried by dimensional reasoning without selecting a specific theory of turbulence.

All of these contributions are valid only for cases where no density gradients exist in the stream. If appreciable differences of density are present, whether because of pressure, temperature, or differences in molecular weight, the problem becomes more difficult. No completely satisfactory solution has yet been offered

- For references, see page 25. 
for the case of a round jet in which gradients of fluid density are appreciable, but the analysis by Abranovitch (10) of the free boundary layer in two-dimensional compressible flow offers hope for this possibility. The recent contribution of Hawthorne, Weddell, and Hottel(11) and the following analysis represent the furthest advance which appears to have been made to date with the problem of an inhomogeneous jet, although neither my properly be regarded as a complete solution. Both require that assumptions be made concerning the profiles of the flow parameters at a cross section to enable the values of these parameters along the axis to be determined. The assumptions made in the treatment presented here appear to be more reasonable for an isothermal jet than those made in the more ambitious attempt by Hawthorne and his co-workers, where the analysis is sufficiently general to consider the effect of combustion. The validity of both works, however, can only be established by comparison with experimental data.

The present status of the problem, therefore, seems to call for further experimental investigation. Frequently, the results of such a study will furnish the clue to a satisfactory analysis. More important, however, is the consideration that, because of the empirical nature of the mixing-length theories of turbulence, the validity of any analytical solution which they might provide can be established only by comparison with experiment. The mean-value relationships provided by these solutions, of course, are not sufficient to explain completely the mixing in a turbulent jet. For this reason, the turbulence itself must be investigated on the basis of the statistical theory. This, by its nature, is primarily an experimental task, best undertaken with hot-wire anemometer. Much study of this sort is currently in progress.

The following section presents an analysis of the flow in a freely expanding jet in which density gradients may exist. The equations of material and momentum balance are written, and the assumption is made that the radial distribution of the velocity and concentration may be approximated by error functions. This assumption makes possible the solution of the equation for the variation of the velocity and the concentration long the axis of the jet.

\section{THEORY OF A FREELY EXPANDING INHOMOGENEOUS JET}

Figure 1 is a sketch of a freely expanding, vertical jet of fluid with density $P_{0}$ issuing from a circular orifice of diameter $D$ with a velocity $u_{0}$ into a medium of density $P_{g}$. Let $x$ denote distances along the axis and $y$ denote radial distances from the axis. The density, the axial component of the velocity, and the concentration of the jet fluid by volume at some point in the stream $(x, y)$ are denoted by $P, u$, and $c$ respectively. 


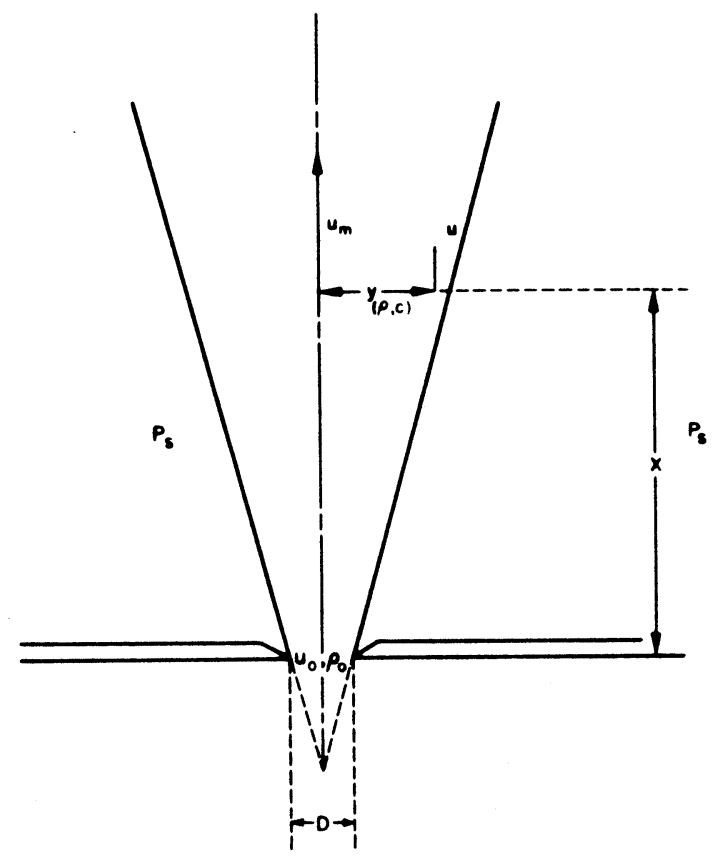

CONFIGURATION OF A FREELY EXPANDING GAS JET

FIG.I

It is assumed that the jet exhibits perfect symmetry of rotation about its axis, and that the pressure gradient in the jet is simply the hydrostatic pressure gradient

$$
\frac{d p}{d x}=-p_{s} g
$$

A material balance of the jet fluid may be written by equating the amount of the fluid which passes any cross section in unit time to the flow rate at the orifice

$$
\begin{gathered}
2 \pi \int_{0}^{\infty} c u y d y=\frac{\pi D^{2} u_{0}}{4} \\
\int_{0}^{\infty} c u y d y=\frac{D^{2} u_{0}}{8} .
\end{gathered}
$$

The momentum flow rate through any cross section is

$$
2 \pi \int_{0}^{\infty} \rho u^{2} y d y .
$$


The difference of the momentum flow rate through any two cross sections which are separated by a distance $d x$ is

$$
2 \pi\left[\frac{d}{d x} \int_{0}^{\infty} \rho u^{2} y d y\right] d x
$$

and is equal to the net force, $F$, which acts on the fluid between the cross sections considered. This force is the resultant of the weight of the fluid and the negative pressure gradient, or, from Eq. (1),

$$
F=\left[2 \pi \int_{0}^{\infty}\left(-\rho_{g}-\frac{d p}{d x}\right) y d y\right] d x=\left[2 \pi_{8} \int_{0}^{\infty}\left(\rho_{s}-\rho\right) y d y\right] d x .
$$

Therefore, the equation of momentum balance ma be written

$$
2 \pi \frac{d}{d x} \int_{0}^{\infty} \rho u^{2} y d y=2 \pi_{g} \int_{0}^{\infty}\left(\rho_{s}-\rho\right) y d y,
$$

and, integrating from the orifice to the cross section,

$$
2 \pi \int_{0}^{\infty} \rho u^{2} y d y=2 \pi g \int_{0}^{x} \int_{0}^{\infty}\left(P_{s}-\rho\right) y d y d x+K_{1} .
$$

where $K_{1}$ is a constant of integration and equal to the momentum flow rate at the orifice,

$$
K_{1}=\frac{\pi D^{2} P_{0} u_{0}^{2}}{4}
$$

so that

$$
\int_{0}^{\infty} \rho u^{2} y d y=\int_{0}^{x} \int_{0}^{\infty}\left(P_{8}-\rho\right) y d y d x+\frac{D^{2} \rho_{0} u_{0}^{2}}{8} .
$$

We now introduce Reynold's representation of the instantaneous values in a turbulent stream by the sum of a mean component and a fluctuating component and denote them by a bar and a prime, respectively.

$$
\begin{aligned}
& u=\bar{u}+u^{\prime} \\
& \rho=\bar{\rho}+\rho^{\prime} \\
& c=\bar{c}+c^{\prime} .
\end{aligned}
$$


In accordance with convention, all mean values of single and triple products of primed components are neglected. Thus, for example,

$$
{\overline{u^{\prime}}}^{\prime}=\overline{\rho^{\prime} u^{\prime} c^{\prime}}=\overline{\rho^{\prime} u^{\prime 2}}=0 \text {. }
$$

Introducing this notation to Eqs. (2) and (3), they become

$$
\int_{0}^{\infty} \bar{c} \bar{y} y d y+\int_{0}^{\infty} \overline{c^{\prime} u^{0}} y d y=\frac{D^{2} u_{0}}{8} \text {. }
$$

and

$$
\int_{0}^{\infty} \bar{\rho} \bar{u}^{2} y d y+\int_{0}^{\infty} \bar{\rho} \overline{u^{\prime 2}} y d y+2 \int_{0}^{\infty} \overline{\rho^{\prime} u^{\prime}} \bar{u} y d y=g \int_{0}^{x} \int_{0}^{\infty}\left(P_{s}-\rho\right) y d y d x+\frac{D^{2} P_{0} u_{0}^{2}}{8} .
$$

Neglecting the terms describing the turbulent transport of momentum and material transport in the $x$ direction, these equations become

$$
\int_{0}^{\infty} \bar{c} \bar{u} y d y=\frac{D^{2} u_{0}}{8} \text {, }
$$

and

$$
\int_{0}^{\infty} \bar{\rho} \bar{u}^{2} y d y=6 \int_{0}^{x} \int_{0}^{\infty}\left(p_{s}-\bar{p}\right) y d y d x+\frac{D^{2} p_{0} u_{0}^{2}}{8}
$$

It is assumed, in accordance with many observations of air jets and those presented below for jets of fluid other than air, that beyond a certain distance from the orifice the profiles of mean concentration and of mean velocity in the jet are similar. Together with the assumption that the edge of the jet is a straight line, this means that these parameters may be expressed as products of functions of the single variables $x$ and $\eta$ equal to $y / x$.

Thus

and

$$
\left.\begin{array}{rl}
\bar{u} & =u_{m}(x) f(\eta) \\
\bar{c} & =c_{m}(x) g(\eta) \\
d y & =x^{2} \eta d \eta
\end{array}\right\}
$$$$
\text { Also, }
$$

Substituting these values into the equation for the material balance of the jet fluid, Eq. (4) becomes

$$
\int_{0}^{\infty} c_{n} u_{n} f(\eta)_{B}(\eta) x^{2} \eta d \eta=\frac{D^{2} u_{0}}{8}
$$


Since no integration with respect to $x$ is performed, functions of $x$ may be taken outside the integral and

$$
c_{m} u_{m} x^{2} \int_{0}^{\infty} f(\eta) g(\eta) \eta d \eta=\frac{D^{2} u_{0}}{8} .
$$

Representing the integral by $I$, and solving for $u_{m}$,

$$
u_{m}=\frac{1}{c_{m} x^{2}} \frac{D^{2} u_{0}}{8 I_{1}} \text {. }
$$

The equation for momentum balance, Eq. (5), is

$$
\int_{0}^{\infty} \bar{\rho} \bar{u}^{2} y d y=8 \int_{0}^{x} \int_{0}^{\infty}\left(p_{s}-\bar{\rho}\right) y d y d x+\frac{D^{2} p_{0} u_{0}^{2}}{8} .
$$

The density is related to the concentration by the equation

$$
\bar{\rho}=p_{s}+\left(p_{0}-p_{s}\right) \bar{c} \text {. }
$$

Substitution from (6) and (11) into Eq.(10) yields

$$
\begin{aligned}
& \rho_{s} u_{m}^{2} x^{2} \int_{0}^{\infty} f^{2}(\eta) \eta d \eta+\left(p_{0}-p_{s}\right) u_{m}^{2} c_{m} x^{2} \int_{0}^{\infty} f^{2}(\eta) g(\eta) \eta d \eta \\
&=-g \int_{0}^{x} c_{m} x^{2} d x \int_{0}^{\infty} g(\eta) \eta d \eta+\frac{D^{2} P_{0} u_{0}^{2}}{8} .
\end{aligned}
$$

Introducing the notation

$$
\left.\begin{array}{l}
I_{1}=\int_{0}^{\infty} f(\eta) \mathrm{g}(\eta) \eta d \eta \\
I_{2}=\int_{0}^{\infty} f^{2}(\eta) \eta d \eta \\
I_{3}=\int_{0}^{\infty} f^{2}(\eta) \mathrm{g}(\eta) \eta d \eta \\
I_{4}=\int_{0}^{\infty} g(\eta) \eta d \eta
\end{array}\right\} .
$$


and substituting for $u_{n}$ from (9), Eq. (12) becomes

$$
\frac{P_{8} D^{4} u_{0}^{2} I_{2}}{64 c_{n}^{2} x^{2} I_{1}^{2}}+\frac{\left(P_{0}-P_{8}\right) D^{4} u_{0}^{2} I_{3}}{64 c_{2}^{2} x^{2} I_{1}^{2}}=-8\left(P_{0}-P_{8}\right) I_{4} \int_{0}^{x} c_{m} x^{2} d x+\frac{D^{2} P_{0} u_{0}^{2}}{8} \text {. }
$$

The term in Eq. (14) containing the integral with respect to $x$ represents the effect of the buoyancy force on the flow. Since this is not a critical term except at very low jet velocities, we may here introduce the approximation,

$$
c_{m} \pm \frac{K D}{x}, \quad K=\text { constant }
$$

so that

$$
\int_{0}^{x} c_{n} x^{2} d x \doteq K D \int_{0}^{x} x d x=\frac{K D x^{2}}{2}
$$

Using this approximation, Eq. (14) becomes

$$
\frac{P_{s} D^{4} u_{0}^{2} I_{2}}{64 c_{m}^{2} x^{2} I_{1}^{2}}+\frac{\left(P_{0}-P_{s}\right) D^{4} u_{0}^{2} I_{3}}{64 c_{m}^{2} x^{2} I_{1}^{2}}=-\frac{x^{2}{ }_{g} K D\left(P_{0}-P_{s}\right) I_{4}}{2}+\frac{D^{2} P_{0} u_{0}^{2}}{8}
$$

Multiplying all terms by the factor $8 c_{m}^{2} x^{2} / D^{4} u_{o}^{2} P_{0}$ and rearranging,

$$
\left[\frac{4 g K D I_{4}\left(P_{0}-P_{8}\right)}{P_{0} u_{0}^{2}}\left(\frac{x}{D}\right)^{4}-\left(\frac{x}{D}\right)^{2}\right] c_{m}^{2}+\frac{\left(\rho_{0}-\rho_{s}\right) I_{3}}{8 \rho_{0} I_{1}^{2}} \cdot c_{m}+\frac{\rho_{s} I_{2}}{8 \rho_{0} I_{1}^{2}}=0 .
$$

Equation (16) is a quadratic in $c_{\text {n }}$ which may be solved easily by the quadratic formula. Before writing this solution, however, consider the integrals $I_{1}, I_{2}, I_{3}$, and $I_{4}$.

Taylor, Grimmett, and Comings (12) have shown that for a jet of air mixing with surrounding air, the approximation

$$
f(\eta) \doteq e^{-k \eta^{2}}
$$

is quite good. Hawthorne, in addition, has shown that for an air jet mixing with air,

$$
\boldsymbol{g}(\eta)=[f(\eta)]^{\mu}
$$

as demonstrated in Appendix I. Assuming that these approximations also may be valid for other jets, then

$$
\begin{aligned}
& f(\eta)=e^{-k \eta^{2}} \\
& B(\eta)=e^{-\mu k \eta^{2}} .
\end{aligned}
$$


Observing that

$$
\int_{0}^{\infty} e^{-b x^{2}} x d x=\frac{1}{b} \int_{0}^{\infty} e^{-y^{2}} y d y=\frac{1}{2 b}
$$

the integrals of (13) may be evaluated readily, as

$$
\left.\begin{array}{l}
I_{1}=\int_{0}^{\infty} e^{-(\mu+1) k \eta^{2} \eta d \eta=\frac{1}{2 k(\mu+1)}} \\
I_{2}=\int_{0}^{\infty} e^{-2 k \eta^{2}} \eta d \eta=\frac{1}{4 k} \\
I_{3}=\int_{0}^{\infty} e^{-(\mu+2) k \eta^{2} \eta d \eta=\frac{1}{2 k(\mu+2)}} \\
I_{4}=\int_{0}^{\infty} e^{-\mu k \eta^{2} \eta d \eta=\frac{1}{2 \mu k}}
\end{array}\right\} .
$$

Substitution from (18) into (16) gives

$$
\left[\frac{2 g K D\left(p_{0}-p_{s}\right)}{\mu P_{0} u_{0}{ }^{2} k}\left(\frac{x}{D}\right)^{4}-\left(\frac{x}{D}\right)^{2}\right] c_{m}^{2}+\frac{\left(p_{0}-p_{s}\right)(\mu+1)^{2} k}{4 p_{0}(\mu+2)} c_{k}+\frac{p_{s} k(\mu+1)^{2}}{8 p_{0}}=0 .
$$

Let ting

and

$$
B \equiv \frac{\left[\frac{\left(\rho_{0}-\rho_{s}\right)(\mu+1)^{2} k}{4 \rho_{0}(\mu+2)}\right]}{\left[\frac{2 g K D\left(p_{0}-p_{s}\right)}{\mu k p_{0} u_{0}^{2}}\left(\frac{x}{D}\right)^{4}-\left(\frac{x}{D}\right)^{2}\right]}
$$

$$
A \equiv \frac{\left[\frac{p_{s} k(\mu+1)^{2}}{8 p_{0}}\right]}{\left[\frac{2 g K D\left(p_{0}-p_{s}\right)}{\mu k p_{0} u_{0}^{2}}\left(\frac{x}{D}\right)^{4}-\left(\frac{x}{D}\right)^{2}\right]},
$$

Eq. (19) becomes

$$
\begin{aligned}
& c_{n}^{2}+B c_{a}+A=0 \\
& c_{n}=\frac{-B \pm \sqrt{B^{2}-4 A}}{2} .
\end{aligned}
$$


The positive root is obviously the one desired, so that

$$
c_{\mathrm{n}}=\frac{1}{2}\left(\sqrt{B^{2}-4 A}-B\right) \text {. }
$$

The axial velocity is obtained from Eq. (9),

$$
\frac{u}{u_{0}}=\frac{k(\mu+1)}{4 c_{k}} \frac{1}{\left(\frac{x}{D}\right)^{2}} \text {. }
$$

Values of $\mu$ and $k$ must be determined from the experimental data for the crosssectional profiles of the velocity and concentration.

\section{EXPERIMENTAL INVESTIGATION}

\section{Experimental Apparatus and Procedure}

Measurements have been made of the values of mean concentration and of mean velocity in jets of helium, nitrogen, and carbon dioxide. All of the jets studied issued from a sharp-edged orifice of 0.128 -inch diameter at a velocity of $400 \mathrm{ft} / \mathrm{sec}$. The mean concentration was determined by analysis of samples drawn from the stream at a slow and constant rate through a hypodermic sampling tube, using a conventional Orsat apparatus. Impact pressures, measured through the same tube and indicated on an inclined manometer, furnished the additional data required to determine the mean velocity of the stream.

Figure 2 is a schematic sketch of the experimental apparatus. The jet fluid leaves the storage cylinder through a pressure regulator and passes through 12 feet of rubber hose to straight section of tubing which terminates in the orifice. This tube is mounted on movable carriage that can be positioned accurately by a micrometer drive, while the sampling probe is supported in a fixed position above it. Static pressure is measured upstream of the orifice at a piezometer ring.

The line from the hypodermic sampling probe leads to a selector valve by which it may be connected to an inclined manometer for measurement of the total pressure, or to the rack of mercury-filled gas pipettes into which samples are drawn and stored for analysis. A reservoir above the rack supplies mercury to displace air in the capillary tubing making up the manifolds and to fill the pipettes. When the orifice is in the desired position beneath the probe, the sampling line is purged by drawing a small volume of gas into a pipette assigned for this purpose, and the sample is then drawn into another pipette by allowing the mercury to flow from it to the lower reservoir. A manometer attached to the sampling line allows at least a comparative estimate of the rate of sampling. This was found, however, to have no detectable effect on the composition of the sample obtained over a large variation in the rate of sampling. 


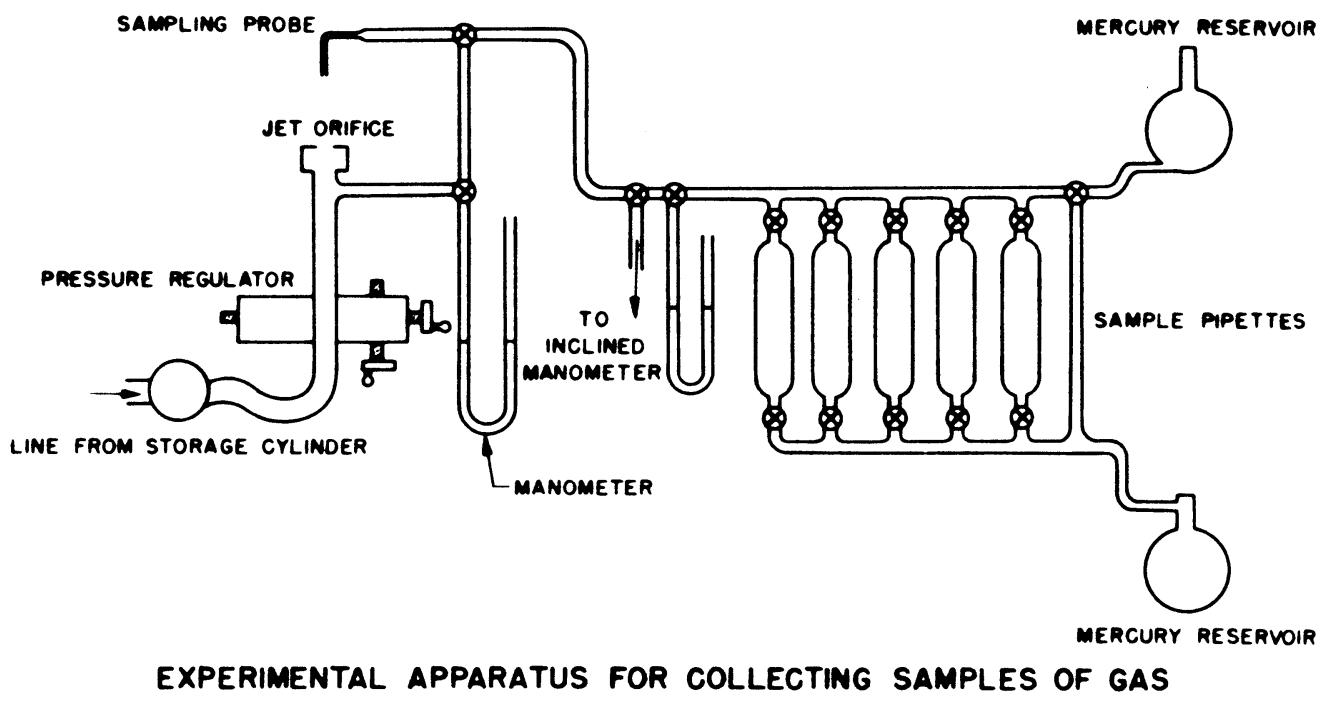

FIG. 2

As noted, the samples were analyzed in a conventional Orsat apparatus. Samples from jets of helium and of nitrogen were analyzed for oxygen, from which the percentage of air in the sample was computed. Those drawn from jets of carbon dioxide were analyzed directly for that gas.

Figure 3 shows the orifice tube, the orifice, and the hypodermic sampling tube in detail.

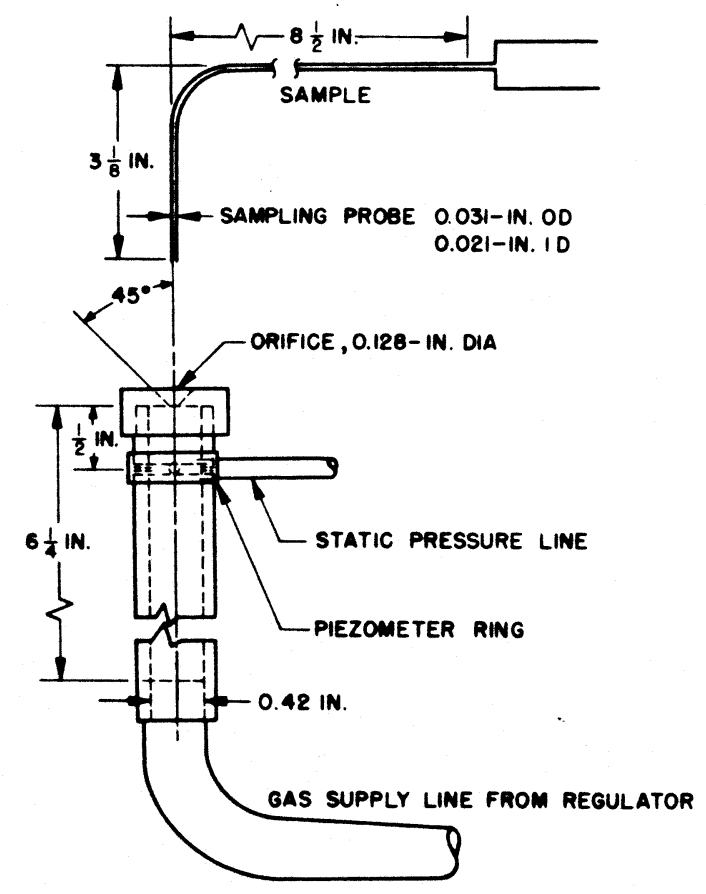

DETAILS OF ORIFICE AND SAMPLING PROBE

FIG.3 


\section{Experimental Data}

Values of the mean concentration of jet fluid and of the mean velocity along the axis were measured in jets of helium, nitrogen, and carbon dioxide. In addition, the cross-sectional profiles of mean concentration mere measured at cross sections located 8,16 , and 24 orifice diameters domstream from the orifice. Velocity profiles were measured at these cross sections in jets of nitrogen and carbon dioxide, but those for a jet of helium were not obtained because of the inadequate sensitivity of the inclined manometer to the low values of impact pressure. In all jets, the velocity at the orifice was $400 \mathrm{ft} / \mathrm{sec}$, and the orifice diameter was 0.128 inch.

Figures 4 and 5 present the measured profiles of concentration and velocity in jets of nitrogen and carbon dioxide measured at cross sections 16 and 24 orifice diameters downstream. These are compared with the error functions which describe these profiles. Values of $\mu$ and $k$ were selected so that the approximation would coincide with the data at the value of $\eta$ where

$$
\frac{c}{c_{m}}=0.5 \text {. }
$$

This gives, for carbon dioxide,

$$
\begin{aligned}
& \mu=0.84 \\
& k=101 ;
\end{aligned}
$$

and for nitrogen,

$$
\begin{aligned}
& \mu=0.72 \\
& k=88 .
\end{aligned}
$$
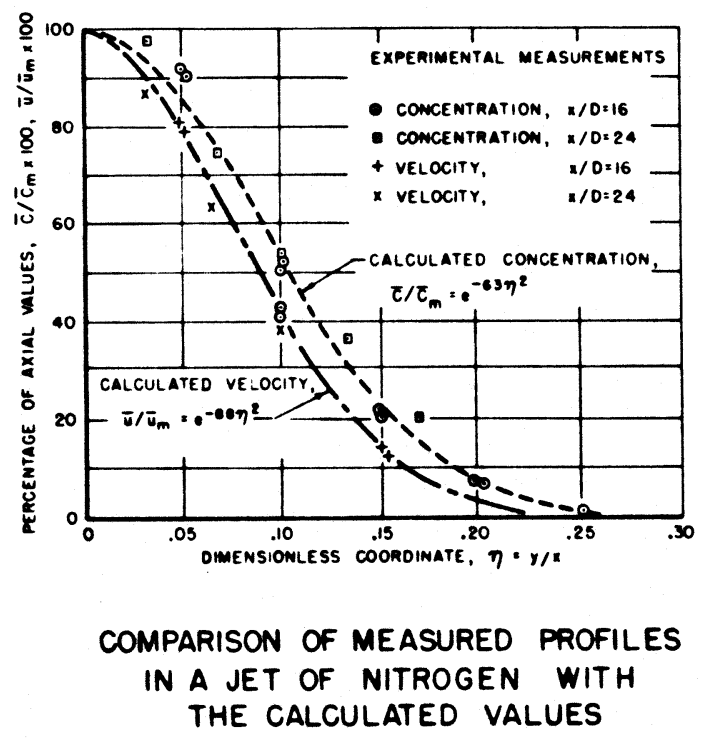

FIG. 4

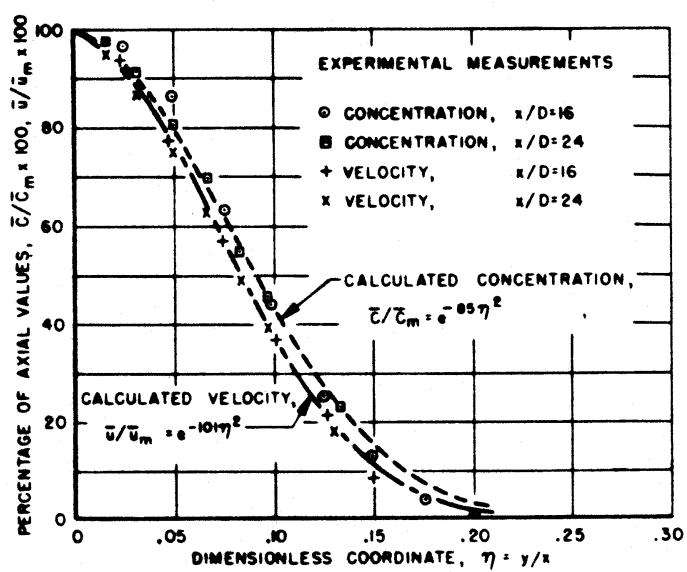
COMPARISON OF MEASURED PROFILES IN A JET OF CARBON DIOXIDE WITH THE CALCULATED VALUES

FIG. 5 
Figure 6 shows the concentration profile in a jet of helium measured at cross sections 16 and 24 orifice diameters downstream. The error function describing this profile is again made to coincide with the data at the value of $\eta$ where

$$
\frac{c}{c_{m}}=0.5 \text {, }
$$

from which, by Eq. (17),

$$
\mu k=28.5 \text {. }
$$

Since the velocity profile was not measured in the jet of helium, values of $\mu$ and $k$ may not be determined separately as for jets of nitrogen and carbon dioxide. It becomes necessary, therefore, to assume a value for one of these. If it is assumed, as a first approximation, that $\mu$ is a linear function of the fluid density, the value of $\mu$ for helium is obtained by extrapolation from those found for carbon dioxide and nitrogen. This gives

$$
\mu \doteq 0.5 \text {, }
$$

and

$$
k \doteq 57 \text {. }
$$

Figure 6 includes the approximation which this assumption gives for the velocity profile.

The data shown in Figs.4, 5, and 6 are taken from cross sections at 16 and 24 orifice diameters downstream from the orifice. In all cases, the profiles of both concentration and velocity are similar at the two cross sections when plotted as shown. Data taken from the cross section 8 orifice diameters downstream are not included, but they were found not to be similar to those shown. This is to be expected from the description of turbulent jets as given by Kuethe. (4)

Figures 7,8 , and 9 compare the values of concentration and velocity measured along the axis of jets of helium, nitrogen, and carbon dioxide with those calculated from Eqs. (20) and (21). The calculations are based on values of $\mu$ and $k$ determined from the cross-sectional profiles as shown. 


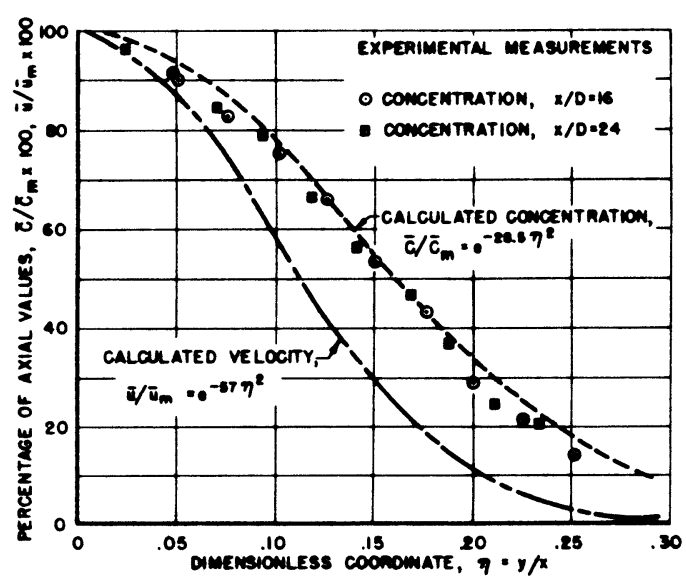

COMPARISON OF MEASURED PROFILES IN A JET OF HELIUM WITH THE CALCULATED VALUES

FIG. 6

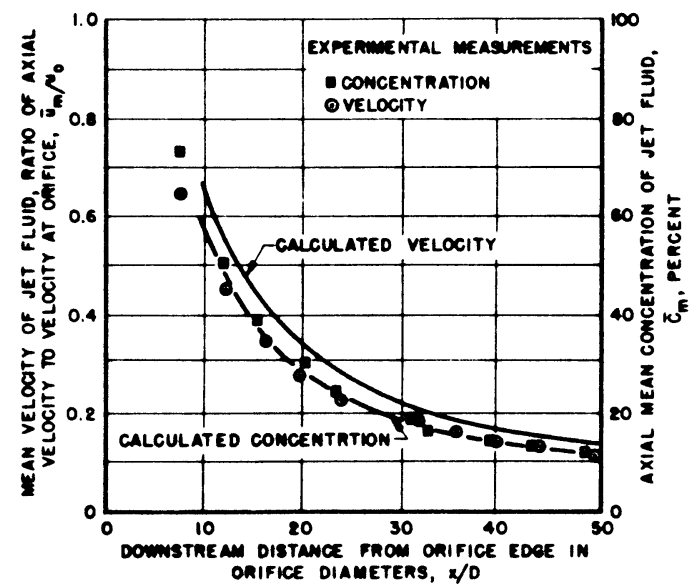

COMPARISON OF MEASURED AXIAL CONCENTRATION AND VELOCITY IN A JET OF NITROGEN WITH THE CALCULATED VALUES

FIG. 7

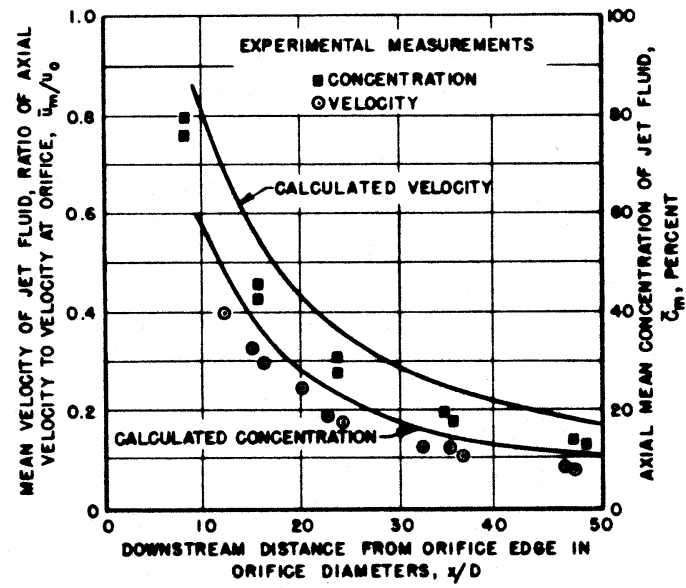

COMPARISON OF MEASURED AXIAL CONCENTRATION AND VELOCITY IN

A JET OF CARBON DIOXIDE WTH THE CALCULATED VALUES

FIG. 8

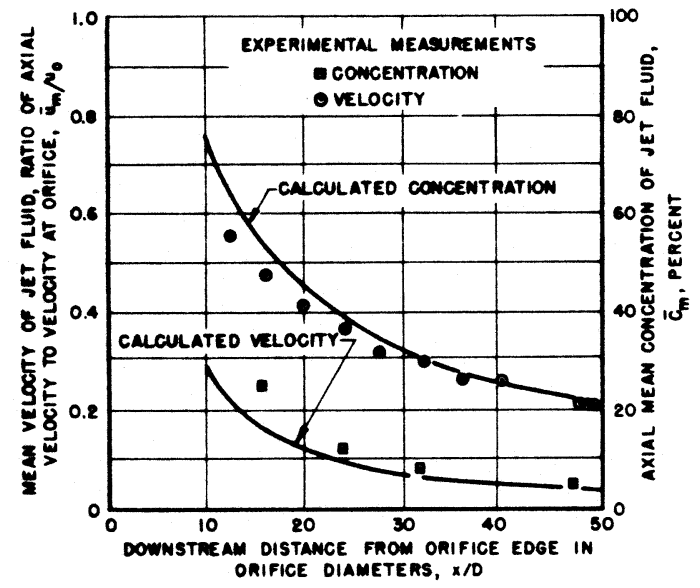

COMPARISON OF MEASURED AXIAL CONCENTRATION AND VELOCITY IN A JET OF HELIUM WITH THE CALCULATED VALUES

FIG. 9 


\section{DISCUSSION AND CONCLUSIONS}

The data reported are, admittedly, of an exploratory nature and may be subject to several consistent errors associated with the experimental techniques. Some indication of the magnitude of these may be gained by a comparison of the data from the jet of nitrogen with the established results for jets of air obtained by other experimenters.

Taylor, Grimmett, and Comings(12) have recently measured velocities in a jet of air and have found the ratio of axial velocity to orifice velocity to be given by

$$
\frac{u_{n}}{u_{0}}=\frac{6.2}{\left(\frac{x}{D}\right)} \text {. }
$$

These are about five percent higher than the data presented in Fig. 8 . From the velocity profiles measured by Taylor and his co-workers, $k$ is 91.5 . This agrees well with the value of 88 determined from Fig.5. By Eqs. (20) and (21), however, Taylor's value of $k$ may be shown to give

$$
\frac{u_{n}}{u_{0}}=\frac{6.7}{\left(\frac{x}{D}\right)},
$$

or approximately six percent higher than his measured values. Taylor observes this discrepancy as an apparent momentum loss in the jet, occurring within few orifice diameters of the nozzle opening.

A similar loss was noted in the data presented above. Values of mean concentration were multiplied by those of the mean velocity and integrated graphically over different cross sections. The integral, according to Eq. (4), should be constant for all cross sections and equal to the flow rate at the orifice divided by $2 \pi$. The calculation, however, performed both for jets of nitrogen and of carbon dioxide, did not give this result but rather indicated an apparent loss of jet fluid quite similar to the momentum loss observed by Taylor.

Because of the limited data on this effect, any attempt to explain it at present would be in the nature of surmise. It should be pointed out, however, that the apparent loss of jet fluid and of momentum may be accounted for by local pressure gradients or by turbulent transfer of matter and momentum parallel to the axis. In the latter case, the terms

$$
\int_{0}^{\infty} \overline{c^{\prime} u^{\prime} y} d y
$$


and

$$
2 \int_{0}^{\infty} \overline{c^{\prime} u^{\prime} \bar{u} y} d y+\int_{0}^{\infty} \bar{c} \overline{u^{12}} y d y
$$

cannot be considered to vanish as in the derivation of Eqs. (4) and (5).

The model used for the analysis of the jet differs from reality in yet another respect by assuming that the concentration and velocity profiles are similar at all cross sections and that the jet originates from a point cource. Actually, the data indicate that such a model approximates a physical jet quite well beyond a certain distance, roughly 10 orifice diameters from the orifice. The virtual point source of the jet, however, need not coincide with the actual orifice but may be displaced from it by several orifice diameters.

The calculated values of velocity and of concentration presented in Figs.7, 8, and 9 are valid only for regions of the jet in which no apparent loss of momentum or jet fluid occurs, and in which the concentration and velocity profiles are similar at all cross sections. If an apparent loss of momentum or jet fluid does occur in the system, the effect will be to displace the curves along the ordinate, and if the virtual point source does not coincide with the orifice, the effect will be to displace the curves along the abscissa. Unfortunately, the data were not considered to be of sufficient accuracy to justify any attempt to calculate the se displacements. An arbitrary adjustment of the curves to conform with the experimental data, on the other hand, would have little technical value.

As a result of the work reported above, the following conclusions are drawn:

1. The cross-sectional profiles of mean concentration and mean velocity in inhomogeneous gas jets exhibit the same type of similarity as do those of bomogeneous gas jets. These profiles may be closely'approximated by appropriate error functions. This conclusion should be further confirmed by data of appreciably greater reliability than those obtained here.

2. The analytic approximation of these profiles permits an algebraic solution of the equations of material and momentum balance to give expressions for the mean concentration and mean velocity along the axis of the jet.

3. Apparent material and momentum loss, due either to pressure gradients or to turbulent transfer parallel to the axis, is observed in the data of others as well as in those reported here. This effect should be further investigated.

4. The effect of buoyancy is predicted by the expressions for the axial concentration and velocity. At high velocities this effect is negligible, so that the prediction cannot be verified by the present data. Further investigation of this effect would be of interest. 



\section{A P P E N D I X I}

\section{THE THEORY OF A HOMOGENEOUS JET*}

If the density is constant throughout the jet, Eqs. (4) and (5) become

$$
\begin{aligned}
& \int_{0}^{\infty} \bar{c} \bar{u} y d y=\frac{D^{2} u_{0}}{8} \\
& \int_{0}^{\infty} \bar{u}^{2} y d y=\frac{D^{2} u_{0}^{2}}{8} .
\end{aligned}
$$

Substitution according to (6) gives

$$
\begin{gathered}
u_{m} c_{m} x^{2} \int_{0}^{\infty} f(\eta) g(\eta) \eta d \eta=\frac{D^{2} u_{0}}{8} \\
u_{m}^{2} x^{2} \int_{0}^{\infty} f^{2}(\eta) \eta d \eta=\frac{D^{2} u_{0}^{2}}{8},
\end{gathered}
$$

whence

$$
\begin{aligned}
& u_{m}=\frac{K_{1}}{x} \\
& c_{m}=\frac{K_{2}}{x},
\end{aligned}
$$

and

$$
\begin{aligned}
& \bar{u}=\frac{K_{1}}{x} f(\eta) \\
& \bar{c}=\frac{K_{2}}{x} g(\eta) .
\end{aligned}
$$

-The analysis presented here is taken from the work of Tollmien(1) and Hawthorne (6). 
Now the equation of continuity is

$$
\frac{\partial \bar{u}}{\partial x}+\frac{1}{y} \frac{\partial}{\partial y}(y \bar{v})=0,
$$

or, changing variable,

$$
\frac{\partial \bar{u}}{\partial x}-\frac{y}{x^{2}} \frac{\partial \bar{u}}{\partial \eta}+\frac{1}{x y} \frac{\partial}{\partial \eta}(y \bar{v})=0 .
$$

Substituting from (24),

$$
\begin{aligned}
-\frac{K_{1} f(\eta)}{x^{2}}-\frac{y}{x^{3}} K_{1} f^{\prime}(\eta)+\frac{1}{x y} \frac{\partial}{\partial \eta}(y \bar{v}) & =0 \\
\frac{\partial}{\partial \eta}(y \bar{v}) & =K_{1}\left[\eta f(\eta)+\eta^{2} f^{\prime}(\eta)\right]=K_{1} \eta \frac{\partial}{\partial \eta}[\eta f(\eta)],
\end{aligned}
$$

and integrating,

$$
\bar{v}=K_{1}\left[\frac{\eta f(\eta)}{x}-\frac{1}{x \eta} \int f(\eta) \eta d \eta\right] .
$$

Letting

$$
\begin{aligned}
& \boldsymbol{F}=\boldsymbol{F}(\eta) \equiv \int f(\eta) \eta d \eta \\
& \bar{u}=K_{1} \frac{F^{\prime}}{x \eta} \\
& \left.\bar{v}=K_{1}\left[\frac{F^{\prime}}{x}-\frac{F}{x \eta}\right]\right\} \text {. }
\end{aligned}
$$

For a homogeneous jet, the Navier-Stokes equation of motion in Cartesian coordinates is

$$
\bar{u} \frac{\partial \bar{u}}{\partial x}-\bar{v} \frac{\partial \bar{u}}{\partial y}=-\overline{v^{\prime} \frac{\partial u^{\prime}}{\partial y}},
$$

when external forces, pressure gradients, and viscosity terms are neglected. In variables $x$ and $\eta$ this becomes

$$
\bar{u} \frac{\partial \bar{u}}{\partial x}-\bar{u} \frac{y}{x^{2}} \frac{\partial \bar{u}}{\partial \eta}+\bar{v} \frac{1}{x} \frac{\partial \bar{u}}{\partial \eta}=-\overline{v^{\prime} \frac{\partial u^{\prime}}{\partial y}} .
$$


Substituting for $\bar{u}$ and $\bar{v}$ from (27),

$$
K_{1}^{2}\left[\frac{F^{\prime} F}{x^{3} \eta^{3}}-\frac{F^{\prime 2}-F^{\prime \prime} F}{x^{3} \eta^{2}}\right]=-\overline{v^{\prime} \frac{\partial u^{\prime}}{\partial y}}
$$

Using Taylor's mixing length, $l_{T}$, we write

$$
\begin{aligned}
& u^{\prime}=\ell_{T} \frac{\partial \bar{u}}{\partial y} \\
& v^{\prime}=\ell_{T} \frac{\partial \bar{u}}{\partial y},
\end{aligned}
$$

and assume

$$
\ell_{T}=c x
$$

Hence

$$
\begin{aligned}
v^{\prime} \frac{\partial u^{\prime}}{\partial y} & =c^{2} x^{2} \frac{\partial \bar{u}}{\partial y} \frac{\partial^{2} \bar{u}}{\partial y^{2}} \\
\frac{\partial \bar{u}}{\partial y} & =\frac{K_{1}}{x^{2} \eta^{2}}\left[F^{\prime \prime} \eta-F^{\prime}\right] \\
\frac{\partial^{2} \bar{u}}{\partial y^{2}} & =\frac{K_{1}}{x^{3} \eta^{3}}\left[F^{\prime \prime \prime} \eta^{2}-2 F^{\prime \prime} \eta+2 F^{\prime}\right] .
\end{aligned}
$$

Equation (28) thus becomes

$$
\frac{F^{\prime} F}{x^{3} \eta^{3}}-\frac{F^{\prime 2}+F^{\prime \prime} F}{x^{3} \eta^{2}}=-\frac{c^{2}}{x^{3} \eta^{5}}\left[\left(F^{\prime \prime} \eta-F^{\prime}\right)\left(F^{\prime \prime \prime} \eta^{2}-2 F^{\prime \prime} \eta+2 F^{\prime}\right)\right],
$$

and this simplifies to

$$
\frac{\partial}{\partial \eta}\left(\frac{F F^{\prime}}{\eta}\right)=\frac{c^{2}}{2} \frac{\partial}{\partial \eta}\left[\frac{\left(F^{\prime \prime}-\frac{F^{\prime}}{\eta}\right)^{2}}{\eta}\right]
$$

whence

$$
F F^{\prime}=\frac{c^{2}}{2}\left(F^{\prime \prime}-\frac{F^{\prime}}{\eta}\right)^{2}
$$


This is the equation for which Tollmien obtained an approximate solution, and the solution is shown in Table 1 .

Table 1

TOLLMIEN'S SOLUTION FOR THE

VELOCITY PROFILE IN A FREE JET

\begin{tabular}{l|l|c|c}
\hline$\frac{\eta}{\sqrt[2]{c^{2} / 2}}$ & $\frac{\bar{u}}{u_{n}}$ & $\frac{\eta}{\sqrt[3]{c^{2} / 2}}$ & $\frac{\bar{u}}{u_{n}}$ \\
\hline 0 & 1.00 & 1.50 & 0.376 \\
0.0625 & 0.995 & 1.75 & 0.283 \\
0.125 & 0.977 & 2.00 & 0.198 \\
0.25 & 0.941 & 2.25 & 0.130 \\
0.375 & 0.895 & 2.50 & 0.077 \\
0.5 & 0.843 & 2.75 & 0.039 \\
0.625 & 0.789 & 3.00 & 0.014 \\
0.75 & 0.727 & 3.25 & 0.002 \\
1.00 & 0.606 & 3.40 & 0.000 \\
1.25 & 0.487 & & \\
\hline
\end{tabular}

For a homogeneous jet, the conservation of jet fluid requires that

$$
v \cdot(\overline{c v})=0 \text {, }
$$

or

$$
\begin{aligned}
\frac{\partial}{\partial x}(\bar{c} \bar{u})+\frac{1}{y} \frac{\partial}{\partial y}(y \bar{c} \bar{v}) & =-\frac{1}{y} \frac{\partial}{\partial y}\left(y \overline{c^{\prime} v^{\prime}}\right) . \\
\frac{\partial}{\partial x}(\bar{c} \bar{u})-\frac{y}{x^{2}} \frac{\partial}{\partial \eta}(\bar{c} \bar{u})+\frac{1}{y x} \frac{\partial}{\partial \eta}(y \bar{c} \bar{v}) & =-\frac{1}{y} \frac{\partial}{\partial y}\left(\overline{y c^{\prime} v^{\prime}}\right) .
\end{aligned}
$$

Substitution from (24), (25), and (27) yields

$$
-K_{1} K_{2}\left\{\frac{2 F_{g}^{\prime} g(\eta)}{x^{3} \eta}+\frac{y}{x^{4}} \frac{\partial}{\partial \eta}\left[\frac{F^{\prime} g(\eta)}{\eta}\right]\right\}+\frac{K_{1} K_{2}}{y x} \frac{\partial}{\partial \eta}\left\{\left[\frac{F^{\prime}}{x}-\frac{F}{x \eta}\right] \eta_{g}(\eta)\right\}=-\frac{1}{y} \frac{\partial}{\partial y}\left(y c^{\prime} v^{\prime}\right) .
$$

Introducing Taylor's mixing length again, write

$$
\begin{aligned}
& c^{\prime}=h_{T} \frac{\partial \bar{c}}{\partial y} \\
& v^{\prime}=h_{T} \frac{\partial \bar{u}}{\partial y} \\
& h_{T}=c x
\end{aligned}
$$




$$
\begin{aligned}
\frac{1}{y} \frac{\partial}{\partial y}\left(\overline{y c^{\prime} v^{\prime}}\right) & =\frac{1}{y} \frac{\partial}{\partial y}\left[c^{2} x^{2} y \frac{\partial \bar{c}}{\partial y} \frac{\partial \bar{u}}{\partial y}\right] \\
& =\frac{c^{2} x^{2}}{x y} \frac{\partial}{\partial \eta}\left[\eta \frac{\partial \bar{c}}{\partial \eta} \frac{1}{x} \frac{\partial \bar{u}}{\partial \eta}\right] \\
& =\frac{c^{2} K_{1} K_{2}}{x^{2} y} \frac{\partial}{\partial \eta}\left[\frac{g^{\prime}(\eta)}{\eta}\left(F^{\prime \prime} \eta-F^{\prime}\right)\right] .
\end{aligned}
$$

Substituting (32) in (31),

$$
\frac{\partial}{\partial \eta}\left[\left(\boldsymbol{F}^{\prime}-\frac{\boldsymbol{F}}{\eta}\right) \eta_{\boldsymbol{B}(\eta)}\right]-2 \boldsymbol{F}^{\prime} \boldsymbol{g}(\eta)-\eta^{2} \frac{\partial}{\partial \eta}\left(\frac{\boldsymbol{F}^{\prime} \boldsymbol{B}(\eta)}{\eta}\right)=-\boldsymbol{e}^{2} \frac{\partial}{\partial \eta}\left[\frac{\boldsymbol{g}^{\prime}(\eta)}{\eta}\left(\boldsymbol{F}^{\prime \prime} \eta-\boldsymbol{F}^{\prime}\right)\right]
$$

and integrating,

$$
\begin{gathered}
\left(F^{\prime} \eta-F\right) g(\eta)-2 \int F_{B}^{\prime}(\eta) d \eta-\eta F^{\prime} B(\eta)+2 \int F^{\prime} g(\eta) d \eta=-c^{2} g^{\prime}(\eta)\left(F^{\prime \prime}-\frac{F^{\prime}}{\eta}\right) \\
F \eta_{B}(\eta)=c^{2}\left(\eta F^{\prime \prime}-F^{\prime}\right) g^{\prime}(\eta) .
\end{gathered}
$$

Dividing (33) by (30),

$$
\begin{aligned}
& \frac{F \eta_{g}(\eta)}{F F^{\prime}}=\frac{c^{2}\left(F^{\prime \prime}-\frac{F^{\prime}}{\eta}\right) \eta g^{\prime}(\eta)}{\frac{1}{2} c^{2}\left(F^{\prime \prime}-\frac{F^{\prime}}{\eta}\right)^{2}} \\
& \frac{\eta_{g}(\eta)}{F^{\prime}}=\frac{2 \eta g^{\prime}(\eta)}{\left(F^{\prime \prime}-\frac{F^{\prime}}{\eta}\right)} .
\end{aligned}
$$

Now

$$
\begin{aligned}
f(\eta) & =\frac{F^{\prime}}{\eta} \\
f^{\prime}(\eta) & =\frac{1}{\eta}\left(F^{\prime \prime}-\frac{F^{\prime}}{\eta}\right)
\end{aligned}
$$

so that

$$
\frac{g(\eta)}{f(\eta)}=\frac{2 g^{\prime}(\eta)}{f^{\prime}(\eta)}
$$


Integrating,

$$
\ln g(\eta)=\frac{1}{2} \ln f(\eta)
$$

The constant of integration is zero, since

$$
f(\eta)=g(\eta)=1
$$

when

$$
\eta=0
$$

Hence

$$
\frac{\bar{c}}{\bar{c}_{m}}=\left(\frac{\bar{u}}{\bar{u}_{m}}\right)^{\frac{1}{2}}
$$





\section{REFERENCES}

(1) Tollmien, W., "Berechnung turbulenter Ausbreitungsvorgänge," Zeit. f. ang. Math. u. Mech., Vol.6 (1926).

(2) Tomotika, S., "Application of the Modified Vorticity Transport Theory to the Turbulent Spreading of a Jet of Air," Proc. Roy. Soc. (London), Vol.165 A (1938), pp.65-72.

(3) Taylor. G.I., "The Transport of Vorticity and Heat Through Fluids in Turbulent Motion," Proc. Roy. Soc. (London), Vol.135 A, p.685.

(4) Kuethe, A.M., "Investigations of the Turbulent Mixing Regions Formed by Jets," J. Applied Mechanics, Vol.2 (1935), pp.87-95.

(5) Squire and Trouncer, R \& M No. 1974 (7591), ACR Technical Report (British Report).

(6) Hawthorne, W.H., "The Mixing of Gas and Air in Flames," Doctorate Thesis (unpublished), M. I.T., Cambridge, Mass., April 1939.

(7) Swain, L.M., "On the Turbulent Wake Behind a Body of Revolution," Proc. Roy. Soc. (London), Vol.125 A (1929), pp.647-659.

(8) Schlichting, H., "Ueber das ebene Windschattenproblem," Ing.-Archiv., Bd. I, Heft 5, 1930.

(8) Squire, H.B., "A Reconsideration of the Theory of Free Turbulence," Phil. Mag., Vol.39, 7 th series, 1948, pp.1-20.

(10) Abramovitch, G.N., The Theory of a Free Jet of a Compressible Gas, NACA Tech. Memo. No.1058, March 1944, p.91.

(11) Hawthorne, W.R., Weddell, D.S., and Hottel, H.C., "Mixing and Combustion in Turbulent Gas Jets" - Paper presented at Third Symposium on Combustion and Flame and Explosion Phenomena, Madison, Wis., September 1948.

(12) Taylor, J.F., Grimmett, H.L., and Comings, E.W., "Flow Characteristics of a Free Jet," Tech. Prog. Report No.2, Fluid Mixing Research, Contract N6-ori-71, T.O. XI, Engineering Experiment Station, University of Illinois, 1948 p.22 (to be published).

(13) Ruden, P., "Turbulente Ausbreitungsvorgänge im Freistrahl," Naturwissenshaften, No.21/23 1933. 